\title{
Parents' attitudes and acceptability of anthropometric measurement of Irish school children
}

\author{
P. M. Heavey ${ }^{1}$, O. McMahon ${ }^{2}$, A. McConnon ${ }^{2}$, U. O'dwyer ${ }^{3}$, C. Hayes ${ }^{4}$, N. Eldin ${ }^{5}$ and C. Kelleher ${ }^{2}$ \\ ${ }^{1}$ Department of Nursing and Health Science, Athlone Institute of Technology, ${ }^{2}$ National Nutrition Surveillance Centre, \\ School of Public Health, Physiotherapy \& Population Science, University College Dublin (UCD), ${ }^{3}$ Department of Health, \\ ${ }^{4}$ Trinity College Centre for Health Sciences and ${ }^{5}$ Health Service Executive, Ireland
}

Measuring children's body size in school is believed to be important in monitoring rates of overweight and obesity. However, measuring body size is likely to be a sensitive issue, even in school-aged children and the acceptance of regular measurement of primary school children to parents, teachers, principals and the children themselves has not been widely examined. In addition, uptake and acceptance, by parents, is central to success of a childhood obesity surveillance programme. One such programme in Ireland is the World Health Organisation European Childhood Obesity Surveillance Initiative (WHO COSI).

As part of the WHO COSI, children from first and third class had their height, weight and waist circumference measured by Nutritionists in the school setting. To date, three rounds of measurements have been completed in 2008, 2010 and 2012. The aim of this research was to investigate what parents think and how they feel about having their children's height, weight \& waist measurements taken in school and their experiences of the WHO COSI exercise. Focus group methodology was used to investigate this issue.

The focus groups $(n=14)$ took place in 14 schools that had previously participated in the WHO COSI. Between 4 and 12 parents attended and these were mostly mothers'. Five fathers' were present at four different focus groups. The sample, determined at the school level, included schools from different socio economic backgrounds and urban and rural areas within the target population. Parents of both boys and girls who had been measured and also parents of children who were not measured were included. Written consent was obtained from the parents. Focus groups were held at the participating schools and lasted about 50 minutes. They were exploratory and based on a topic guide that allowed questioning that was responsive and provided an opportunity for parents to express their opinions and views. One researcher attended all groups and the discussions were digitally recorded and transcribed verbatim. Thematic analysis methodology was used to analyse the transcripts.

Overall parents were aware of increasing prevalence of childhood overweight and obesity and identified many factors that are known to contribute to it. Most parents felt that school played an important role in educating children about healthy eating and providing an environment to engage in physical activity. Although parents were generally positive about measuring children in the school environment, they were concerned about privacy, embarrassment issues, bullying, increased likelihood of eating disorders and dieting. Overall, parents were aware of the WHO COSI exercise and were satisfied with the information provided and they reported positive feedback from their children regarding the exercise. However, parents would prefer if they were provided with feedback regarding results. The findings from this study suggest that measuring children in school is acceptable to parents, as long as it is undertaken in a private setting and they appreciate the importance of carrying out these measurements. 